\title{
CUIDADOS PALIATIVOS: ENFOQUE NO CUIDADO DE ENFERMAGEM À FAMÍLIA
}

\author{
PALLIATIVE CARE: FOCUS IN FAMILY NURSING CARE
}

\section{CUIDADOS PALIATIVOS: ENFOQUE EN LA ATENCIÓN DE ENFERMERÍA FAMILIAR}

\begin{abstract}
Ana Paula Santana da Silva ${ }^{1}$, Andressa Santos de Souza² ${ }^{2}$ Zenaide Leopoldina Luísa Santana Santos Silva ${ }^{3}$, Maria Aparecida de Araújo Silva ${ }^{4}$, Maiara Rodrigues dos Santos ${ }^{5}$
\end{abstract}

\begin{abstract}
RESUMO
Introdução: A assistência de pacientes em cuidados paliativos ainda encontra desafios na incorporação de práticas voltadas ao cuidado centrado na família. Objetivo: Caracterizar a literatura científica sobre cuidado de enfermagem à família de pacientes em cuidados paliativos. Método: Estudo de revisão integrativa de literatura realizado no portal da Biblioteca Virtual de Saúde entre junho e setembro de 2019. Resultados: A análise integrou 10 publicações que abordam o cuidado de enfermagem à família de pacientes em cuidados paliativos, destacando as seguintes categorias: fortalecimento da comunicação e estratégias de manejo familiar, promoção de assistência integral, garantia de redes de suporte, fortalecimento da Atenção Primária, cuidado com a qualidade de vida dos familiares e no atendimento domiciliar, apoio para as decisões de final de vida. Conclusão: Embora haja carência de referenciais teóricos de família para embasar as pesquisas, este estudo apresenta uma análise entre os conceitos do cuidado centrado na família e as evidências sobre assistência de enfermagem à família de pacientes em cuidados paliativos.
\end{abstract}

DESCRITORES: Enfermagem; Família; Cuidados Paliativos; Enfermagem Familiar.

\begin{abstract}
Introduction: Patient care in palliative care still faces challenges in incorporating family-centered care practices. Objective: To characterize the scientific literature on family nursing care in palliative care. Method: Integrative literature review study conducted between June and September 2019. Results: The analysis integrated 10 publications which addresses nursing care to the family of patients in palliative care, highlighting the following categories: strengthening communication and family management strategies, promotion of comprehensive care, guaranteeing support networks, strengthening Primary Care, caring for family members' quality of life and home care, support for end-of-life decisions. Conclusion: Although there is a lack of theoretical family references to support the researches, this study presents an analysis between the concepts of family-centered care and nursing care to the family of patients in palliative care.
\end{abstract}

DESCRIPTORS: Nursing; Family; Palliative Care; Family Nursing.

\section{RESUMEN}

Introducción: la atención al paciente en cuidados paliativos aún enfrenta desafíos para incorporar prácticas de atención centradas en la familia. Objetivo: caracterizar la literatura científica sobre cuidados de enfermería a la familia de pacientes en cuidados paliativos. Método: estudio de revisión de literatura integral realizado en el portal de la Biblioteca Virtual de Salud entre junio y septiembre de 2019. Resultados: el análisis integró 10 publicaciones que abordan la atención de enfermería a la familia de pacientes en cuidados paliativos, destacando las siguientes categorías: fortalecimiento estrategias de comunicación y gestión familiar, promoción de la atención integral, garantía de redes de apoyo, fortalecimiento de la atención primaria, cuidado de la calidad de vida de los miembros de

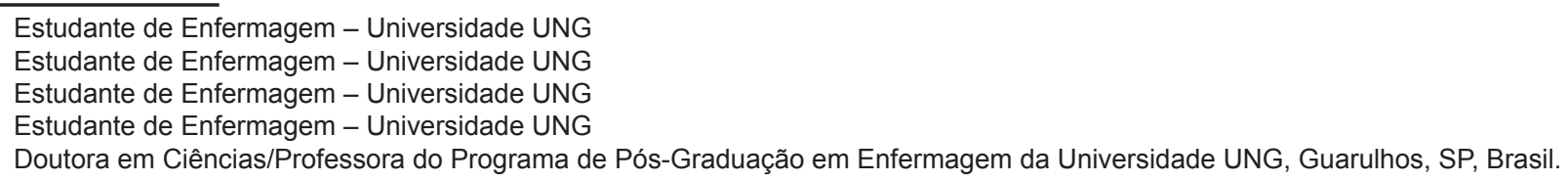


la familia y atención domiciliaria, apoyo para las decisiones al final de la vida. Conclusión: aunque faltan referencias familiares teóricas para apoyar la investigación, este estudio presenta un análisis entre los conceptos de cuidado centrado en la familia y cuidado de enfermería para la familia de pacientes en cuidados paliativos.

DESCRIPTORES: Enfermería; Familia; Cuidados Paliativos; Enfermería de la Familia.

\section{INTRODUÇÃO}

Em 2002, a OMS (Organização Mundial da Saúde) definiu cuidados paliativos como uma abordagem para melhoria da qualidade de vida de pacientes, adultos ou crianças e suas famílias que enfrentam doenças que ameaçam a vida, com o propósito de prevenir e aliviar o sofrimento, por meio da identificação precoce, avaliação adequada e tratamento da dor e outros sintomas ${ }^{1}$. Os cuidados paliativos envolvem assistência integral de pacientes e familiares com doenças que ameaçam à vida, sejam elas crônicas, degenerativas, neurológicas e, especialmente em programas de atenção a idosos².

Uma das características dos cuidados paliativos é a atenção integral, que considera a condição física do paciente, o corpo doente e também sintomas de ordem psicossocial e espiritual. Além disso, os cuidados paliativos incluem assistência à família, que pode ser considerada elemento fundamental na abordagem centrada na pessoa ou como unidade de cuidado na abordagem centrada na família. Embora os modelos de cuidado centrado na família tenham demonstrado impacto enorme na saúde e no bem-estar dos indivíduos, ainda há muitos desafios para garantir sua eficácia em cuidados paliativos ${ }^{3}$.

De acordo com o Instituto de Cuidados Centrados no Paciente e na Família (Institute for Patient-and Family-Centered Care) o cuidado centrado no paciente e na família pode ser definido como "uma abordagem para o planejamento, a entrega e a avaliação dos cuidados de saúde que se baseiam em parcerias mutuamente benéficas entre os profissionais de saúde, os pacientes e as famílias. Essa perspectiva baseia-se no reconhecimento de que pacientes e famílias são aliados essenciais para a qualidade e a segurança no cuidado - não apenas nas interações diretas, mas também na educação dos profissionais de saúde, pesquisa, projeto de instalações e desenvolvimento de políticas. $\mathrm{O}$ atendimento centrado no paciente e na família leva a melhores resultados de saúde, melhor experiência de cuidado do paciente e da família, melhor satisfação do clínico e do pessoal e alocação mais inteligente dos recursos"4.
Para incorporação de tais conceitualizações no plano de cuidado de pacientes em cuidados paliativos no Brasil, recentemente foi publicado a Resolução $N^{\circ} 41$, de 31 de outubro de 2018, que dispõe sobre as diretrizes para organização dos cuidados paliativos no âmbito do Sistema Único de Saúde (SUS). Tal dispositivo representa um avanço para ampliar a oferta de cuidados paliativos no sistema de saúde brasileiro e garante que cuidados apropriados sejam oferecidos à família, com assistência interdisciplinar, como parte integrante dos cuidados paliativos ${ }^{5}$.

Para a assistência à família nos cuidados paliativos é fundamental que a equipe de saúde tenha conhecimento da melhor forma de abordar esses familiares, respeitando seus momentos de indagações, auxiliando nos processos de mudanças em sua estrutura familiar, decorrentes do adoecimento, da perda e morte de seus entes queridos ${ }^{2}$.

Sendo a família a principal fonte de amparo e de cuidado do paciente, o cuidado à família é necessário visto que os processos de fim de vida envolvem relevante impacto social, pela restrição da autonomia dos indivíduos e pelas possíveis mudanças na dinâmica econômica e psicossocial da família, afetando não apenas a vida do paciente, como daqueles que o cercam ${ }^{6}$.

Diante dos desafios para integrar o cuidado centrado na família, enquanto modelo teórico, na organização e sistematização da assistência dos cuidados paliativos, propomos este estudo a partir da seguinte questão norteadora: Como a literatura científica aborda os cuidados de enfermagem à família de pacientes em cuidados paliativos? Assim, o objetivo deste estudo foi caracterizar a literatura científica sobre cuidado de enfermagem à família de pacientes em cuidados paliativos.

\section{MÉTODO}

Tratou-se de uma revisão integrativa de literatura, que possui caráter analítico e descritivo, pois busca apresentar resultados qualitativos a partir da busca sistemática e ordenada da literatura, visando abranger e 
elucidar o conhecimento sobre o tema proposto, a partir do método Prática Baseada em Evidência (PBE). Foi utilizado o modelo do cuidado centrado na família como referencial teórico para a análise ${ }^{4}$. Possui seis etapas: definição de hipótese ou questão de pesquisa, critérios para inclusão ou exclusão de pesquisa, categorização dos estudos, avaliação dos estudos incluídos e interpretação de resultados ${ }^{7}$.

Este estudo teve como questão norteadora: "Como a literatura científica aborda os cuidados de enfermagem à família de pacientes em cuidados paliativos?". Para tal, o acrônimo PICO foi adaptado para pesquisas não clínicas ${ }^{8}$, considerando então o paciente $(P)$, a Intervenção (I) e o contexto (Co) que especificamente para esta pesquisa foram: família $(P)$, cuidado de enfermagem (I) e cuidados paliativos (Co), respectivamente. A inserção das palavras nas bases de dados seguiu essa mesma orientação para definição dos descritores que compuseram as estratégias de busca, conforme mostra o Quadro 1.

\begin{tabular}{|c|c|c|c|}
\hline \multicolumn{4}{|c|}{$\begin{array}{l}\text { BUSCA (combinação de descritores (DECS) e } \\
\text { palavras chaves) }\end{array}$} \\
\hline$P$ & Paciente/problema & $\begin{array}{l}\text { (Família OR Saúde da } \\
\text { Família OR Relações } \\
\text { Profissional-Família OR } \\
\text { Enfermagem Familiar) }\end{array}$ & AND \\
\hline I & $\begin{array}{l}\text { Intervenção ou } \\
\text { Fenômeno de } \\
\text { Interesse }\end{array}$ & \begin{tabular}{|c|} 
(cuidados de enfermagem \\
OR Processo de \\
Enfermagem OR \\
Planejamento de Assistência \\
ao Paciente OR enfermagem)
\end{tabular} & AND \\
\hline Co & Contexto (Co) & $\begin{array}{c}\text { (Cuidados Paliativos OR } \\
\text { Enfermagem de Cuidados } \\
\text { Paliativos na Terminalidade } \\
\text { da Vida) }\end{array}$ & AND \\
\hline \multicolumn{4}{|c|}{$\begin{array}{c}\text { Como a literatura científica aborda os cuidados de } \\
\text { enfermagem à família de pacientes em cuidados } \\
\text { paliativos? }\end{array}$} \\
\hline
\end{tabular}

Quadro 1: Cruzamento de descritores norteado pela estratégia PICO

O levantamento bibliográfico foi realizado nos meses de junho e setembro de 2019, no portal da Biblioteca Virtual de Saúde (BVS), que inclui as fontes de informações das bases de dados: Literatura Latino-Americana e do Caribe em Ciências da Saúde (LILACS), Índice Bibliográfico Español en Ciencias de la Salud (IBECS); Medical Literature Analysis and Retrieval System Online (MEDLINE) e Biblioteca Cocharane.
Os descritores selecionados para realizar a busca foram os termos do Medical Subject Headings (MESH), também contemplados nos Descritores em Ciências da Saúde (DeCS), são eles: Família/Family, PaliativeCare/ Cuidados paliativos, Nursing Care/Cuidados de enfermagem, a partir dos seguintes cruzamentos: (Família OR Saúde da Família OR Relações Profissional-Família OR Enfermagem Familiar) AND (Cuidados de enfermagem OR Processo de Enfermagem OR Planejamento de Assistência ao Paciente OR enfermagem) AND (Cuidados Paliativos OR Enfermagem de Cuidados Paliativos na Terminalidade da Vida).

Os critérios de inclusão foram: pesquisas originais que abordassem ações de enfermagem em cuidados paliativos voltadas às famílias, publicadas nos idiomas português, inglês ou espanhol a partir de 1990, ano em que a OMS padronizou a definição do termo pela primeira vez. Os artigos selecionados foram pesquisas originais com o objetivo de sintetizar evidências científicas sobre a assistência de enfermagem à família de pacientes em cuidados paliativos. Foram excluídas as publicações repetidas, artigos de opinião, monografias, estudos com estudantes e pesquisas realizadas com crianças. Inicialmente, realizou-se a análise de títulos e resumos do estudo. Os artigos selecionados foram lidos na íntegra e analisados a partir da questão norteadora. A figura 1 apresenta detalhadamente as etapas de seleção de artigos para análise.
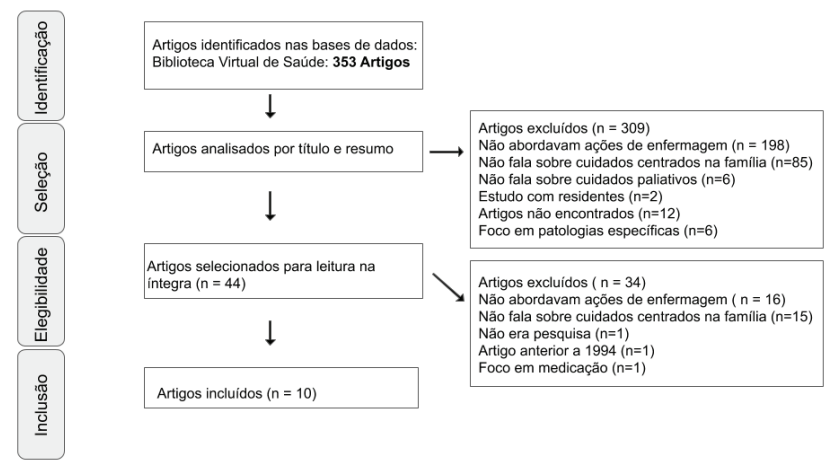

Figura 1: Fluxograma da etapa de seleção do material para análise dos dados.

\section{RESULTADOS}

Os estudos foram selecionados e classificados segundo os critérios de inclusão. A partir da busca foram encontrados 353 títulos pelo portal BVS (Biblioteca Virtual da Saúde) dos 353 textos, 44 foram selecionados para leitura na íntegra e apreciação de conteúdo. Fo- 
ram excluídos 34 textos e, data forma, a amostra final foi constituída de 10 artigos para análise de acordo com o objetivo da pesquisa, ano e país da publicação, desenho do estudo, população e amostra, principais resultados e conclusões. O quadro 2 apresenta uma síntese das principais características dos estudos e os objetivos.

A exploração teórica na literatura resultou em 10 publicações ${ }^{9-18}$. Os estudos foram publicados entre os anos de 2004 e 2019, obtendo-se maior volume de publicações em $2018^{10,12}$. Quanto ao local de origem da realização dos estudos, observou-se diversos países, com predomínio no Brasil com quatro estudos ${ }^{9,10,12,13}$ e Estados Unidos da América (EUA) com três estudos ${ }^{14,15,18}$. Demais locais envolveram a Inglaterra ${ }^{16}$, a China $^{17}$ e Cingapura ${ }^{11}$. No que se refere aos participantes desses estudos, em oito deles, havia profissionais de outras áreas ${ }^{7,11,13,14,17,21-23}$.

Quadro 2 - Caracterização dos artigos pesquisados.

\begin{tabular}{|c|c|c|c|}
\hline Autor/País/Ano & Tipo de Estudo & Objetivo & Unidade amostral \\
\hline $\begin{array}{l}\text { Andrade GB, Pedroso } \\
\text { VSM, Weykamp JM, et } \\
\text { al./Brasil/2019 }{ }^{9}\end{array}$ & $\begin{array}{l}\text { Revisão da } \\
\text { literatura }\end{array}$ & $\begin{array}{l}\text { Conhecer e analisar a produção científica no período } \\
\text { de } 2005 \text { à } 2016 \text { em relação cuidados paliativos e } \\
\text { a importância da comunicação na estratégia dos } \\
\text { cuidados paliativos. }\end{array}$ & $\begin{array}{l}14 \text { artigos } \\
\text { científicos }\end{array}$ \\
\hline $\begin{array}{l}\text { Pessalacia JDR, Silva } \\
\text { AE, Araújo DHQ et al./ } \\
\text { Brasil/2018 }{ }^{10}\end{array}$ & $\begin{array}{l}\text { Descritivo/ } \\
\text { qualitativo }\end{array}$ & $\begin{array}{l}\text { Identificar e compreender as experiências } \\
\text { vivenciadas pelos cuidadores familiares de pacientes } \\
\text { elegíveis para CP* atendidos na APS\$; Identificar } \\
\text { quais são as redes de suporte disponíveis aos } \\
\text { cuidadores familiares de pacientes elegíveis para CP } \\
\text { atendidos na APS. }\end{array}$ & $\begin{array}{l}20 \text { cuidadores } \\
\text { familiares de } \\
\text { pacientes } \\
\text { elegíveis para CP } \\
\text { cadastrados na } \\
\text { APS. }\end{array}$ \\
\hline $\begin{array}{l}\text { Mabel Q. H. Leow, Sally } \\
\text { W. C. Chan./ EUA//2016 } \\
11\end{array}$ & $\begin{array}{l}\text { Descritivo/ } \\
\text { qualitativo }\end{array}$ & $\begin{array}{l}\text { O objetivo deste estudo foi descrever as } \\
\text { experiências de cuidadores familiares que cuidam de } \\
\text { uma pessoa com câncer avançado em casa. }\end{array}$ & $\begin{array}{l}19 \text { cuidadores } \\
\text { familiares }\end{array}$ \\
\hline $\begin{array}{l}\text { Abreu AISCS, Costa } \\
\text { Júnior ÁL/ Brasil/2018 }{ }^{12}\end{array}$ & $\begin{array}{l}\text { Revisão } \\
\text { integrativa }\end{array}$ & $\begin{array}{l}\text { Identificar as repercussões do cuidar de um paciente } \\
\text { oncológico em Cuidados Paliativos e a importância } \\
\text { da atuação da enfermagem no suporte aos } \\
\text { cuidadores familiares. }\end{array}$ & $\begin{array}{l}14 \text { artigos } \\
\text { científicos }\end{array}$ \\
\hline $\begin{array}{l}\text { Capello EMCS, Velosa } \\
\text { MVM, Salotti SRA, } \\
\text { Guimarães HCQCP/ } \\
\text { Brasil/2012 } 13\end{array}$ & $\begin{array}{l}\text { Transversal / } \\
\text { Exploratório / } \\
\text { Quantitativo e } \\
\text { Qualitativo }\end{array}$ & $\begin{array}{l}\text { Identificar o confronto de pacientes com câncer e } \\
\text { seus familiares/cuidadores diante da terminalidade } \\
\text { da vida }\end{array}$ & $\begin{array}{l}6 \text { pacientes } \\
\text { oncológicos fora } \\
\text { das possibilidades } \\
\text { de cura e } 10 \\
\text { familiares/ } \\
\text { cuidadores }\end{array}$ \\
\hline $\begin{array}{l}\text { Royak-Schaler R, Gadalla } \\
\text { SM, P. Lemkau J,D. Ross } \\
\text { D, Alexander C, Scott D. I } \\
\text { EUA /2006 }{ }^{14}\end{array}$ & $\begin{array}{l}\text { Exploratório / } \\
\text { Qualitativo }\end{array}$ & $\begin{array}{l}\text { Avaliar a comunicação do profissional de saúde } \\
\text { sobre o fim da vida e cuidados paliativos com } \\
\text { pacientes com câncer terminal e suas famílias, na } \\
\text { perspectiva da família membros. }\end{array}$ & $\begin{array}{l}24 \text { cônjuges } \\
\text { e parentes de } \\
\text { pacientes falecidos } \\
\text { com câncer }\end{array}$ \\
\hline $\begin{array}{l}\text { Parker Oliver, Debra./ } \\
\text { EUA /2005 } 15\end{array}$ & $\begin{array}{l}\text { Descritivo/ } \\
\text { Qualitativo }\end{array}$ & $\begin{array}{l}\text { Explorar como os hospitais americanos integram } \\
\text { paciente e família na estrutura e processos das } \\
\text { equipes interdisciplinares. }\end{array}$ & $\begin{array}{l}30 \text { funcionários de } \\
\text { um hospice }\end{array}$ \\
\hline $\begin{array}{l}\text { J. McPherson Christine, } \\
\text { M. Addington-Hall Julia./ } \\
\text { Inglaterra / } 2004^{16}\end{array}$ & $\begin{array}{l}\text { Survey } \\
\text { retrospectivo }\end{array}$ & $\begin{array}{l}\text { Elucidar os processos de decisão de familiares } \\
\text { enlutados durante suas avaliações da dor, ansiedade } \\
\text { e depressão dos pacientes. }\end{array}$ & $\begin{array}{l}30 \text { familiares } \\
\text { enlutados }\end{array}$ \\
\hline $\begin{array}{l}\text { Cui J, Song L.J, Zhou L.J, } \\
\text { et al/ China / } 2014^{17}\end{array}$ & $\begin{array}{l}\text { Survey/ } \\
\text { Quantitativo }\end{array}$ & $\begin{array}{l}\text { Explorar as necessidades não atendidas de } \\
\text { cuidadores de pacientes com câncer avançado no } \\
\text { continente chinês e os possíveis fatores associados } \\
\text { a elas. }\end{array}$ & $\begin{array}{l}649 \text { cuidadores } \\
\text { familiares de } \\
\text { pacientes com } \\
\text { câncer. }\end{array}$ \\
\hline $\begin{array}{l}\text { W. Kirk, Timothy./ EUA/ } \\
2007^{18}\end{array}$ & Estudo de caso & $\begin{array}{l}\text { Apresentar um estudo de caso com um plano de } \\
\text { ação para engajar membros familiares de pacientes } \\
\text { com câncer em um serviço de hospital. }\end{array}$ & 1 família \\
\hline
\end{tabular}

${ }^{*} \mathrm{CP}$ (Cuidados Paliativos); ${ }^{\$}$ APS (Atenção Primária à Saúde) 
A análise dos artigos permitiu conhecer ações de cuidado de enfermagem aos familiares de pacientes em cuidados paliativos, esclarecendo as necessidades e demandas da família. Foram elencadas categorias de análise que retratam como a literatura científica aborda o cuidado de enfermagem à família de pacientes em cuidados paliativos e foram relacionadas aos conceitos do cuidado centrado no paciente e na família, sendo elas: fortalecimento de estratégias de comunicação, promoção de assistência integral durante toda a trajetória de doença, garantia de redes de suporte, fortalecimento dos cuidados paliativos na Atenção Primária, cuidado com a qualidade de vida dos familiares, apoio para o cuidado domiciliar, estratégias de manejo familiar e cuidado nas decisões de final de vida. Tais categorias apresentam caráter interde- pendente aos conceitos do modelo de cuidado centrado na família, no entanto, para a análise foi considerado o principal atributo de cada uma das categorias para estabelecer uma relação entre categoria e conceito. O quadro 3 aborda os principais resultados e conclusões encontrados, de acordo com essas categorias de análise.

Entre as principais demandas da família em cuidados paliativos foi identificado o apoio emocional, que pode ser desempenhado pelo profissional de enfermagem integrante da equipe multidisciplinar, mas demanda o desenvolvimento de habilidades para adequado suporte emocional desses familiares. Poucas evidências fortalecem intervenções de cuidados para a família de paciente no contexto paliativo, voltadas às necessidades físicas, psicossociais e espirituais na assistência de enfermagem.

Quadro 3: Categorias de análise sobre cuidado de enfermagem à família de pacientes em cuidados paliativos

\section{CATEGORIAS DE ANÁLISE DESCRIÇÃO DOS PRINCIPAIS RESULTADOS E CONCLUSÕES}

\section{Dignidade e respeito}

Cuidado com a qualidade de As experiências ocasionam estresse devido às intensas emoções e necessidade de vida dos familiares enfrentamento de cuidadores, há intensa sobrecarga e impactos na qualidade de vida do cuidador familiar ${ }^{10,11,13,16,18}$.

Cuidadores experimentam uma ampla gama de emoções negativas como medo, pavor, raiva, culpa, arrependimento, ansiedade, sofrimento, desamparo, fadiga mental e física ${ }^{11,13,14,16}$.

Promoção de assistência integral durante toda a O enfermeiro tem um papel fundamental para a promoção do cuidado paliativo aos familiares, nas fases inicias do diagnóstico e no auxílio para conviver com a doença, prestando trajetória de doença

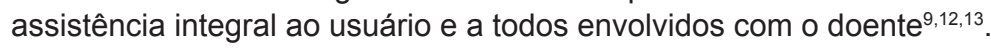

\begin{tabular}{|c|c|}
\hline Compartilhamento de inform & Jes \\
\hline $\begin{array}{l}\text { Fortalecimento de estratégias } \\
\text { de comunicação }\end{array}$ & $\begin{array}{l}\text { Em cuidados paliativos, a relação interpessoal do enfermeiro e do paciente deve considerar } \\
\text { a comunicação como estratégia para fortalecimento do vínculo entre profissional, paciente e } \\
\text { família }^{9,12,18} \text {. }\end{array}$ \\
\hline
\end{tabular}

\section{Participação}

Estratégias de manejo familiar

Identificação e estratégias de manejo para as novas composições na dinâmica familiar, diante da diminuição forçada da jornada de trabalho, problemas financeiros ${ }^{12}$.

Cuidado para decisões de final de vida

Compartilhamento de informações para apoiar as decisões no tratamento e de final de vida, contribuindo para uma compreensão mais adequada ${ }^{16}$.

Familiares não recebem educação sobre cuidados paliativos como uma opção para doenças que ameaçam a vida, antes da tomar decisões sobre os cuidados ${ }^{14}$.

Há necessidade de que todos os membros da equipe de cuidados paliativos apoiem igualmente as decisões dos pacientes e seus familiares ${ }^{15}$.

Manutenção da saúde, apoio para conhecimento sobre a doença e tratamento, apoio no funeral, informações sobre cuidados paliativos para os familiares ${ }^{17}$.

\section{Colaboração}

Garantia de redes de suporte Os cuidadores vivenciam mudanças em suas rotinas diárias, o que pode acarretar sobrecarga física e mental aos mesmos, levando à necessidade de redes de apoio formais e informais. No entanto, inexiste uma rede de apoio formal aos cuidadores e falta treinamento e orientação suficientes para realização do cuidado junto a seu familiar ${ }^{10,12,14}$.

Fortalecimento dos cuidados $\quad$ Ressalta-se a importância da atuação na atenção primária a saúde, por meio do apoio às paliativos na Atenção necessidades biopsicossociais destes cuidadores ${ }^{10,13,17}$.

Primária

Apoio para o cuidado

domiciliar

Capacitação para o atendimento das necessidades destes pacientes no ambiente domiciliar ${ }^{10,18}$. 


\section{DISCUSSÃO}

O presente estudo teve como referencial teórico o modelo do cuidado centrado na família para análise do cuidado enfermagem à família de pacientes em cuidados paliativos. Os conceitos principais deste modelo referem-se: dignidade e respeito - pacientes e familiares são ouvidos e respeitados pelos profissionais de saúde sobre suas perspectivas e escolhas; compartiIhamento de informações - informações claras, objetivas e que facilitem a vida do cuidador e do paciente; participação - incentivar a família no processo de cuidar e na tomada de decisões; colaboração - familiares, paciente e profissionais de saúde trabalhando em conjunto no desenvolvimento, implantação, avaliação de políticas e programas para prestação de cuidados ${ }^{4}$. Esses conceitos foram relacionados a cada uma das categorias de análise dos artigos, mas apresentam caráter interdependente.

O conceito dignidade e respeito, por exemplo, está imbricado em todas as categorias como elemento fundamental para as ações de cuidado centrado na família ${ }^{4}$. A análise da literatura permitiu identificar entre as ações de enfermagem o cuidado com a qualidade de vida dos familiares de pacientes em cuidados paliativos. As estratégias de enfermagem objetivam minimizar o estresse decorrente das emoções negativas e sobrecarga do cuidado e envolvem a promoção do conforto, compreensão do diagnóstico diante do adoecimento, alívio da dor e apoio por meio do diálogo, toque, suporte psicológico, quando necessário. Ainda, faz-se necessário que os enfermeiros desenvolvam habilidades para atuar com empatia, compreensão, respeito e altruísmo, proporcionando bem-estar, segurança e confiança para o paciente e seus familiares ${ }^{9,12,13,16}$.

Os familiares e cuidadores mostraram experimentar uma ampla gama de emoções negativas como medo, pavor, raiva, culpa, arrependimento, ansiedade, sofrimento, desamparo, fadiga mental e física. $O$ foco da assistência de enfermagem é proporcionar o alívio dos principais sintomas estressores, por meio de uma equipe interdisciplinar que apoie e ampare o paciente e sua família até o momento do luto, respeitando suas preferências e decisões durante todo o tratamento ${ }^{11,13,14,16}$.

Outro conceito fundamental do cuidado centrado no paciente e na família é o compartilhamento de informações. Neste estudo, relacionado a este conceito foi identificado o fortalecimento de estratégias de comunicação no cuidado de enfermagem à família de pacientes em cuidados paliativos. Isso porque, a experiência de familiares revelou dificuldades na compreensão das informações fornecidas pelos profissionais de saúde, pelo uso excessivo das terminologias médicas ${ }^{14}$. Tais dificuldades revelam falhas na comunicação que prejudicam o estabelecimento do compartilhamento de informações, tal como preconizado nos conceitos básicos de cuidados centrado na família 4 .

A vivência dos familiares e cuidadores mostra importantes mudanças em suas rotinas diárias o que pode ocasionar uma sobrecarga física e mental aos mesmos, levando à necessidade de redes de apoio formais e informais para estes cuidados, reforço espiritual, físico e emocional. Este suporte pode ser fornecido, em grande parte, pelos profissionais de enfermagem integrantes da equipe multidisciplinar, cujas características da função propiciam um natural vínculo estabelecido com os pacientes e as famílias, permitindo a identificação de fragilidades e o apoio com atitudes, gestos e conhecimento técnico e, até mesmo, com o acionamento de outros integrantes da equipe para tratar de um problema específico ${ }^{10,12,14}$.

A participação que consiste em envolver pacientes e familiares no processo de cuidar e nas tomadas de decisões foi relacionado às categorias de estratégias de manejo familiar e cuidado para as decisões de final de vida. Sabe-se que as famílias de pacientes com doenças que ameaçam à vida enfrentam diversos conflitos para conciliar demandas do cuidado com as necessidades internas e, nesse sentido, o apoio dos profissionais da saúde para manejar a situação e oferecer suporte durante a trajetória de doença é fundamental ${ }^{19}$. Ao mesmo tempo, auxiliá-los nas decisões de final de vida é importante não apenas como forma de aliviar a sobrecarga emocional, mas também para apoiar o luto vivenciado após a perda do familiar ${ }^{20}$.

O sucesso na comunicação entre os profissionais da saúde, o paciente e seus familiares podem promover ou impedir o processo decisório ${ }^{3,9,12,18}$. Além disso, envolve premissas importantes do cuidado centrado na família, como o compartilhamento de informações e a participação ${ }^{4}$.

A colaboração, enquanto um conceito do cuidado centrado na família, pressupõe que familiares, pacientes e profissionais de saúde trabalhem em conjunto no desenvolvimento, implantação, avaliação de políticas e programas para prestação de cuidados ${ }^{4}$. Em relação à colaboração foi identificado pela análise dos dados o fortalecimento das redes de suporte aos familiares de 
pacientes em cuidados paliativos, o que demanda meIhores políticas públicas para assistência da família de pacientes em cuidados paliativos. Outras categorias como a fortalecimento dos cuidados paliativos na Atenção Primária e apoio para o cuidado domiciliar mostram a necessidade de atuar em uma esfera social para a prática do cuidado centrado à família em cuidados paliativos. Ainda é recente no Brasil estratégias consolidadas para a prática dos cuidados paliativos como política pública, sendo que são necessários novos desenhos e programas para a melhoria dos cuidados paliativos, no que se refere tanto ao sistema e aos serviços de saúde quanto às ações na área de assistência social ${ }^{5,21}$.

A literatura analisada mostrou carência de estudos que se apropriem de referenciais teóricos relacionados ao cuidado da família, sendo esta, portanto, a principal contribuição deste estudo. Os estudos não trazem uma definição de família e o termo família e cuidadores, muitas vezes, foram utilizados como sinônimos. Novos estudos de revisão integrativa são necessários para aprofundar a análise da utilização de referenciais teóricos de família no cuidado de enfermagem em cuidados paliativos. Ressaltamos que esta análise foi realizada em apenas uma base de dados, constituindo como a principal limitação deste estudo.

\section{CONCLUSÃO}

Este estudo teve como objetivo caracterizar a literatura científica sobre cuidado de enfermagem à família de pacientes em cuidados paliativos, tendo como referencial teórico os conceitos básicos de cuidados centrado no paciente e na família.

A literatura científica reforça a necessidade da consolidação de melhores alicerces para o relacionamento do profissional com os familiares, permitindo estabelecer confiança, por meio de uma comunicação efetiva, participação das decisões e esclarecimentos de dúvidas.

Os dados analisados integraram diversas intervenções, delineando ações de enfermagem que promovam o cuidado integral, em uma abordagem física, social, psicológica e espiritual. Embora haja uma carência de referenciais teóricos sobre família para embasar as pesquisas, este estudo propôs uma análise das relações entre os conceitos do cuidado centrado na família e as evidências científicas sobre cuidado de enfermagem à família de pacientes em cuidados paliativos. 


\section{REFERÊNCIAS}

WHO: World Health Organization WHO Definition of Palliative Care; 2002 [acesso em 2019 maio 31]. Disponível em: <https://www.who.int/cancer/palliative/definition/en/>

Gomes ALZ, Othero MB. Cuidados paliativos. Estudos Avançados [internet]. 2016 [acesso em 2019 nov 10];30(88):155-166. Disponível em: https://dx.doi. org/10.1590/s0103-40142016.30880011

Kissane DW. O desafio do cuidado centrado na família na medicina paliativa. Ann Palliat Med. 2016; 5(4):319-321.

Johnson, BH, Abraham, MR. Partnering with patients, residents, and families: a resource for leaders of hospitals, ambulatory care settings, and long-term care communities. Bethesda, MD: Institute for Patient- and Family-Centered Care. 2002 [acesso em 2019 nov 10]. Disponível em: <http://www.ipfcc.org/about/pfcc.html>

Brasil. Congresso. Senado. Resolução № 41, De 31 De Outubro De 2018 Diário Oficial da União n 209, de 30 de outubro de 2018, Seção 1, p. 57.

Espíndola AV, Quintana AM, Farias CP, München MAB. Relações familiares no contexto dos cuidados paliativos. Rev bioét. 2018; 26(3):371-7.

Mendes KDS, Silveira RCCP, Galvão CM. Revisão integrativa: método de pesquisa para a incorporação de evidências na saúde e na enfermagem. Texto Contexto Enferm. 2008; 17(4): 758-64.

Santos CMC, Pimenta CAM, Nobre MRC. A estratégia pico para a construção da pergunta de pesquisa e busca de evidências. Rev Latino-am Enfermagem [internet]. 2007 [acesso em 2019 nov 10];15(3):508511. Disponível em: https://dx.doi.org/10.1590/S010411692007000300023

Andrade GB, Pedroso VSM, Weykamp JM, et al. Cuidados paliativos e a importância da comunicação entre o enfermeiro e paciente, familiar e cuidador. Rev Fund Care Online. 2019; 11(3):713-717.

Pessalacia JDR, Silva AE, Araújo DHQ et al. Experiências de cuidadores nos cuidados paliativos e redes de suporte. Rev enferm UFPE. 2018; 12(11):2916-22.
Leow MQ, Chan SW. The challenges, emotions, coping, and gains of family caregivers caring for patients with advanced cancer in singapore. Cancer Nurs. 2017; 40(1):22-30.

Abreu AISCS, Costa Júnior ÁL. Sobrecarga do cuidador familiar de paciente oncológico e a enfermagem. Rev enferm UFPE. 2018; 12(4):976-86.

Capello EMCS, Velosa MVM, Salotti SRA, Guimarães HCQCP. Enfrentamento do paciente oncológico e do familiar/cuidador frente à terminalidade de vida. $\mathrm{J}$ Health Sci Inst. 2012; 30(3):235-40.

Royak-Schaler R, Gadalla S, Lemkau J, Ross D, Alexander $C$, Scott D. Family perspectives on communication with healthcare providers during end-of-life cancer care. Oncol Nurs Forum. 2006; 33(4):753-60.

Parker OD, Porock D, Demiris G, Courtney K. Patient and family involvement in hospice interdisciplinary teams. J Palliat Care. 2005; 21(4):270-6.

McPherson CJ, Addington-Hall JM. Evaluating palliative care: bereaved family members' evaluations of patients' pain, anxiety and depression. J Pain Symptom Manage. 2004 Aug; 28(2):104-14.

Cui J, Song LJ, Zhou LJ, Meng H, Zhao JJ. Needs of family caregivers of advanced cancer patients: a survey in Shanghai of China. Eur J Cancer Care (Engl). 2014 Jul; 23(4):562-9. doi: 10.1111/ecc.12174.

Kirk T. Managing Pain, Managing Ethics. Pain Management Nursing. 2007; 8(1):25-34.

Mooney-Doyle K, dos Santos MR, Szylit R, Deatrick JA. Parental expectations of support from healthcare providers during pediatric life-threatening illness: a secondary, qualitative analysis. J Pediatr Nurs. 2017; 36:163-172

Santos MR, Wiegand DL, Sá NN, Misko MD, Szylit R. From hospitalization to grief: meanings parents assign to their relationships with pediatric oncology professional. Rev Esc Enferm USP. 2019;53:e03521.

Frossard A. Os cuidados paliativos como política pública: notas introdutórias. Cad. EBAPE.BR. 2016; 14(12): 640-655 